\title{
AL MARGEN DE LAS OBRAS DE FRANCISCO DE LA TORRE
}

\author{
SOLEDAD PÉREZ-ABAdín BARRO \\ Universidad de Santiago de Compostela
}

El volumen que en 1631 publica Quevedo con el título de Obras del Bachiller Francisco de la Torre ${ }^{1}$ supone el primer testimonio impreso de la actividad poética de este autor, que hacia 1588 tramitaba la solicitud de autorización para imprimir sus versos, según figura en un registro del Archivo General de Simancas, por el que se concede al autor licencia para imprimir su poesía y privilegio de venta por diez años:

Licencia a Francisco de la Torre, vecino de Salamanca, para imprimir un libro intitulado los versos líricos y adónicos y la bucólica y privilegio para le poder vender por tiempo de diez años (Archivo General de Simancas, Cámara de Castilla, Libros de Relaciones, ${ }^{\circ}{ }^{\circ} 23$, fol. $\left.180 r\right)^{2}$.

Este proyecto, autorizado mediante provisión que el Consejo Real despacha el 19 de marzo de 1588 (fol. 178r), no llegó a materializarse en un libro impreso hasta que Quevedo reanuda el proceso a partir del manuscrito rubricado que llega a su poder. La edición que entonces prepara consta de dos partes claramente diferenciadas: la poesía de Francisco de la Torre, compuesta por cuatro libros, y una antología de traducciones, conocida con el nombre de «apéndice», que llevan a cabo diferentes poetas salmantinos.

\footnotetext{
${ }^{1}$ En Madrid en la Imprenta del Reyno. / Año de M.DC.XXXI. / A costa de Domingo Gonçalez mercader / de libros.

${ }^{2}$ El hallazgo se debe a Rojo Vega, que lo publica en «Manuscritos y problemas de edición en el siglo XVI» (1994: 152). Interpreto este documento en mis trabajos «Un nuevo dato sobre Francisco de la Torre: la real provisión documentada en el Archivo de Simancas», «La poesía de Francisco de la Torre: un proyecto editorial frustrado» y «Nota bibliográfica acerca de Francisco de la Torre». La investigación desarrollada por mí en torno al tema es independiente del artículo de Colón Calderón, 2002.
}

Rlit, LXV, 130 (2003), 375-390 


\section{LAS TRADUCCIONES DEL APÉNDICE}

El tránsito entre ambas secciones se indica en el propio volumen al término de la égloga octava de la Bucólica del Tajo: «Fin de las obras de Francisco de la Torre» (fol. 130r), colofón que da paso a un nuevo núcleo, el conjunto de traducciones que presenta Juan de Almeida en su carta, presididas por el rótulo: SIGVENSE TRADV / ciones de Horacio, y del Petrarca, / del maestro Sanchez / Brocense (fol. 131r).

Si la primera parte situaba al frente de las páginas el título, dividido entre el verso y el recto de los folios: «Obras del Bachiller / Francisco de la Torre», este segundo núcleo dispone de un nuevo epígrafe: «Traducciones del / Maestro Sanchez» (132r-144v). Tales encabezamientos de página funcionan como marca adicional de la separación de los núcleos.

Parecería obvio que el epígrafe Síguense traducciones... que preside el conjunto se debe a Juan de Almeida, que a continuación firma la epístola al lector. Almeida realizaría la compilación de las traducciones y su ensamblaje con los versos de Francisco de la Torre. La primera palabra, Síguense, cumpliría la función de gozne entre ambos núcleos tan dispares entre sí, pero resultado de una concepción unitaria, gracias a la labor editorial de Almeida.

Desde otro punto de vista, el título de la sección podría responder a un estadio posterior y ser obra de quien realizó la conjunción de dos manuscritos poéticos independientes, el de las poesías de La Torre y el de las traducciones presentadas por Almeida. En algún momento de su transmisión, ambos núcleos convergen y así llegan a Quevedo, que justifica su enlace a través del epígrafe Síguense, delator del rango adicional de esta muestra antológica ${ }^{3}$.

En definitiva, el propio epígrafe de las traducciones, según figura en la edición príncipe, aporta indicios de su carácter marginal. Con posterioridad, la crítica aplicaría el término apéndice para definirlo como agregación a las Obras de La Torre. Acerca de ellas, escribe Menéndez Pelayo: «en sus últimas páginas aparece un curioso apéndice, dirigido por D. Juan de Almeida a los lectores» (1885: 27). Comenzaría así a hacer fortuna un término que Zamora Vicente elige para titular el conjunto de traducciones. Este editor distingue la segunda parte del volumen con el título general de Apéndice (1969:179), para, a continuación (1969: 181), copiar el encabezamiento de la editio princeps.

El texto que ofrece Cerrón Puga prescinde de la denominación de apéndice, contraria a la unidad que la editora propone para las dos partes del

\footnotetext{
${ }^{3}$ Blanco considera que el epígrafe «fue un encabezamiento necesario, obligatorio, que tuvo que ser añadido por Quevedo» (1982: 359).
} 
volumen, y reproduce el título de 1631 (1993: 287). Para segmentar este conjunto de composiciones, introduce dos epígrafes, que faltan en la editio princeps: Traducciones del Maestro Francisco Sánchez el Brocense (1993: 291-308) y Traducciones de Fray Luis de León, el Maestro Francisco Sánchez el Brocense, Don Juan de Almeida y Alonso de Espinosa (1993: 309-316). De esta forma da cierto orden a lo que en definitiva constituye un agregado dispar de versiones poéticas.

Abre la sección un fragmento en prosa, a modo de epístola al lector, en el que Juan de Almeida se presenta como responsable de la edición del conjunto de traducciones entre las que también se incluyen algunas del Brocense:

Don Iuan de Almeida, a quien lee.

AViendo comunicado estos versos con el Maestro Francisco Sanchez de las Broças, Catedratico de propiedad de Retorica de la Vniversidad de Salamanca, de cuyas buenas, y singulares letras tanta noticia y opinion se tiene, no solo en España, pero en las mas principales partes de Italia, y Francia. $\mathrm{Y}$ teniendo tambien conocimiento de algunas traduciones suyas: con cuyo raro trabajo auia adornado algunos sonetos de Francisco Petrarca, y otras Odas de Horacio, medroso de ver estos papeles sin ornamento de algun escritor deste tiempo, le supliqué los pusiesse juntamente con ellos: con cuya autoridad no dudo sino q iran seguros al juyzio de los hombres sabios» (fol. 131r).

En el prólogo de Almeida falta una referencia explícita a Francisco de La Torre y a sus poemas. Esta omisión representa una primera anomalía, indicio de una génesis independiente de ambas secciones, convergentes en una fase posterior. Tal vez Quevedo encontró ambos manuscritos previamente ensamblados o él mismo decidió su unión, justificada con el epígrafe Síguense...

Las menciones de «estos versos» y «estos papeles» no contradicen esta autonomía, pues en principio nada inclina a interpretarlas como alusivas a las poesías precedentes sino que parecen anticipar los textos a los que la carta de Almeida sirve de presentación. La antología recopila las versiones realizadas por el Brocense de algunas odas de Horacio (II-10, I-5), de sonetos de Petrarca (XV, XVIII, CLXI, XIX, XLVIII, CLXXXIX, LXIV, CCLXXII, CXXXII, CXXXIV, CLXXII) y de un soneto de Domenico Veniero. Tales serían las aportaciones solicitadas por Almeida, que expresa el deseo de que sus propias traducciones, y las traducciones ajenas reunidas por él, salgan a la luz con el aval que proporciona el prestigio de Sánchez de las Brozas. De ahí su alegación final: «medroso de ver estos papeles sin ornamento de algun escritor deste tiempo, le supliqué los pusiesse juntamente con ellos: con cuya autoridad no dudo sino q iran seguros al juyzio de los hombres sabios» (fol. 131r).

Atendiendo a su autoría, las traducciones se reparten en diferentes gru- 
pos. El primero comprende las odas trasladadas por Sánchez de las Brozas (fols. 132r-138r), a quien también se atribuye por error Por que te das tormento (Quid fles, Asterie, III-7), realizada por Fray Luis de León ${ }^{4}$ (fols. $138 r-139 v$ ). Siguen las tres versiones que de la oda $O$ navis (I-14) llevan a cabo Almeida, Sánchez y Espinosa (fols. 139v-141v) y, tras unas líneas de transición que comienzan «Auiendo traduzido tres tan grandes poetas...», se disponen las cartas intercambiadas entre estos y Fray Luis de León, que a su vez aporta su propia traducción de la oda $O$ navis. Constituye este conjunto una sección unitaria, que circuló como obra suelta, según documentan varios testimonios manuscritos ${ }^{5}$. Sirve de cierre un comentario sobre las cisuras o encabalgamiento léxico, que aunque se asignó alguna vez a Almeida parece ser del Brocense ${ }^{6}$.

En apariencia, las traducciones de $O$ navis podrían identificarse con los versos o papeles comunicados por Almeida con el Brocense, a pesar de que este mismo se halla entre los traductores de la oda. La contribución de Fray Luis, por lo demás, hacía innecesaria la solicitud de «ornamento de algun escritor deste tiempo». Tales objeciones se oponen a este primer significado de la carta preliminar y sugieren su desconexión de la antología de traducciones que pretende introducir.

Sostener una lectura alternativa de la carta, identificando «estos versos» y «estos papeles» con las Obras de Francisco de la Torre, comporta nuevas dificultades. Las referencias elusivas al poeta carecen de justificación, salvo si se admite que la epístola reproduce sólo un fragmento de un texto más amplio, del que se ha perdido un inicio que hacía mención explícita del poeta ${ }^{7}$. Surge entonces un reparo fundamental, derivado de la falta de congruencia cronológica de los datos de que se dispone. El año de la muerte de Almeida ${ }^{8}, 1572$, ofrece un límite ante quem de composición de la carta, anterior al acceso del Brocense a los títulos de catedrático de

\footnotetext{
${ }^{4}$ Menéndez Pelayo (1885: 28) indica que la oda se debe a Fray Luis, «entre cuyas poesías se ha impreso siempre».

${ }^{5}$ Biblioteca del Palacio Real (ms. 1580, fol. 56), Biblioteca del Palacio Real (ms. 531, fol. 240), Biblioteca Colombina (ms. 82-3-39, 93), Biblioteca Pública de Sevilla (ms. 613). Reproducidos y estudiados por Blanco Sánchez en el Apéndice II de su libro (1982: 479-490). Cfr. Cerrón, 1984: 312.

${ }^{6}$ Cerrón (1984: 53-56) demuestra que se debe al Brocense y está copiado de las anotaciones que añadió Ruscelli a I Fiori delle Rime. Venecia, 1558.

${ }^{7}$ Hughes (1982: 10-11) opina que la carta está incompleta, pues la imprecisa referencia a «estos versos» resulta abrupta. En opinión de Alatorre (1999: 62-70), el sintagma «estos versos», que aparece en la carta inicial y se repite en el comentario sobre la $\mathrm{ci}$ sura, remite en el primer caso a Fray Luis y en el segundo a La Torre.

${ }^{8}$ Según Coster (1925: 107), se produciría entre el 24 de marzo de 1572 y el 5 de febrero de 1573. Sena (1974: 69-77) sitúa el año de nacimiento del teólogo en el lustro de 1530-35. Blanco (1982: 55) enmarca la vida de Juan de Almeida entre 1542 y el 13 de agosto de 1572.
} 
Retórica y de Maestro ${ }^{9}$ y a la fecha de la primera aprobación de Ercilla, en 1579 , según ha sido notado por la crítica. Se suma ahora el dato de la fecha de la autorización del Consejo Real concedida a Francisco de la Torre en 1588. Carece de sentido, por lo tanto, sostener que Almeida se hallaba en posesión del manuscrito de La Torre y que a él se refería en su carta introductoria, cuando el propio poeta, quince años más tarde, se encarga personalmente de los trámites de impresión.

La hipótesis pierde consistencia a la luz del permiso para imprimir de 1588. De ello también se desprende la evidencia de que la unión de los textos de La Torre y las traducciones del apéndice, preparadas por Almeida o por el Brocense, se produjo después de este año ${ }^{10}$.

Por las razones cronológicas ya expuestas, si la carta fue escrita por Juan de Almeida, los versos y papeles en ella aludidos no pueden identificarse con la poesía de $\mathrm{La}$ Torre. Si por el contrario tales referencias apuntan a las Obras, debe rechazarse la autoría de Almeida del fragmento preliminar, obra del artífice del ensamblaje de ambos manuscritos. La carta cumpliría entonces una función de nexo similar al epígrafe y podría atribuirse al mismo autor del título Síguense... Quevedo, por lo tanto, adquiere un original con dos conjuntos de textos previamente unidos a través de esos goznes si no es que el propio editor, al percatarse de su heterogeneidad, decide intercalar una transición que sirva de enlace, atribuyéndosela a Almeida.

Dentro de este supuesto, cobra coherencia la vinculación de los preámbulos de Quevedo a la poesía de Francisco de la Torre y la epístola inicial del apéndice, partícipes de un común sentido equívoco sobre la supuesta antigüedad de un autor que se trata de actualizar con escritos contemporáneos. En sus prefacios a las Obras, Quevedo pone en duda su inicial ase-

\footnotetext{
${ }^{9}$ En la edición de 1631 estos títulos no sólo aparecen en la carta, sino en los encabezamientos de página ya señalados: Traducciones del /Maestro Sanchez (título y nombre en el recto del folio). El Brocense es nombrado Catedrático de Retórica en 1573 y Maestro en 1574, como ya notó Crawford (1927: 369-370). Tal vez accedió al cargo de Catedrático con anterioridad o bien él mismo introdujo esa corrección en el título, según propone Blanco (1982: 354). Cerrón considera que el cambio de titulación académica de «Maestro» a «Catedrático» ocurre cuando el Brocense pasa a ser el poseedor del manuscrito, después de la muerte de Almeida (1984: 56-57).

${ }^{10}$ Para Cerrón, corresponde al Brocense el mérito de la preparación de la antología: «todo el apéndice, excepto la página de presentación hecha por Almeida, habría sido preparado por él, y por esta razón se reproduciría en su Opera omnia y en los demás manuscritos en los que se encuentra» (1984: 56). Existen dudas acerca de la autoría de los distintos elementos: una oda de Fray Luis se atribuye por error al Brocense; el comentario sobre el encabalgamiento podría deberse a Almeida, pero parece ser obra del Brocense, que lo traduce de las anotaciones que añadió Ruscelli a I Fiori delle Rime. Venecia, 1558 (1984: 53-56). Blanco (1982: 393-399) sugiere que Quevedo retocó el apéndice y que las versiones horacianas y las de los sonetos de Petrarca y Veniero son de Fray Luis y no de Sánchez (1982: 360-385).
} 
veración de que se trata de un autor anterior a Boscán, aduciendo en contra «el propio razonar suyo tan bien pulido con la mejor lima destos tiempos» y «la grandeza de su estilo», despojado de arcaísmos (fol. $8 r-v$ ). El prólogo de Don Iuan de Almeida, a quien lee justifica la solicitud de traducciones del Brocense: «medroso de ver estos papeles sin ornamento de algun escritor deste tiempo, le supliqué los pusiesse juntamente con ellos». Esta referencia del apéndice remite a la idea expuesta por Quevedo en sus preámbulos y parece sugerir si no su autoría, al menos una intervención manipuladora, encaminada a conectar sendos preliminares.

A tenor de esta lectura, el propósito actualizador del editor no deja de ser paradójico, ya que estas traducciones compiladas por Almeida serían anteriores a 1572, de modo que precederían en algo más de una década a los versos de La Torre, suponiendo la inmediatez de fechas de la composición de estos y del inicio de los trámites legales para publicarlos.

FRANCISCO DE LA TORRE EN EL CONTEXTO POÉTICO CONTEMPORÁNEO: EL CÍRCULO SALMANTINO Y HERRERA

Como se ha intentado demostrar, la edición de Quevedo de 1631 reúne en un único volumen dos conjuntos literarios autónomos, encabezados por sus propios prólogos, entre sí también diferentes a pesar de algunos elementos de enlace que quizá fueron interpolados para contrarrestar la disparidad de las unidades conjugadas. La inusual duplicación de textos preliminares apunta a la independencia de cada una de las secciones que encabezan, que en origen no habrían sido concebidas para circular juntas, en forma manuscrita o impresa.

Por otra parte, sorprende que los textos que reproducen las disposiciones legales, al frente del libro impreso, no mencionen el epílogo de traducciones, sin referencia en las aprobaciones de Vander Hammen y de Valdivielso. En sus preámbulos Quevedo pasa por alto estas páginas finales y por el contrario repara en otros pormenores del original por él editado, tales como la palinodia de la primera hoja, Delirabam cum hoc faciebam... (fol. 15v), la aprobación de Ercilla y las rúbricas del Consejo (fols. $7 v-8 r)$. Si la carta introductoria del apéndice aludía a las Obras de La Torre, sería esperable que Quevedo comentase este intercambio de versos entre Almeida y el Brocense, a fin de justificar su relación con el conjunto precedente y aportar un dato más acerca del enigmático poeta. La omisión de cualquier comentario parece indicar que las páginas del apéndice se adosan como añadido azaroso y marginal, que Quevedo da a la imprenta probablemente porque así ha llegado el original a sus manos.

La teoría, apuntada por Blanco Sánchez, de que este apéndice figurase como prólogo en un estadio anterior resuelve en parte su carácter 
anómalo ${ }^{11}$. Cumpliría el cometido de las poesías laudatorias ajenas ${ }^{12}$ que forman parte de los textos preliminares dispuestos tras la portada e incorporados después de que la obra recibe la autorización legal. Contradice esta explicación la ausencia de referencias apologéticas al autor y, sobre todo, la extensión y factura irregular de la antología.

El apéndice, en efecto, surge de una urdimbre heterogénea de textos, que comprende traducciones en verso y pasajes de crítica literaria acerca del arte de traducir o sobre una licencia poética. Aunque Quevedo no haya sido el artífice de su unión con las poesías de Francisco de la Torre, ni el autor de la transición, título y carta, entre ambas secciones, resulta innegable que este elenco de traducciones de autores salmantinos reforzaba su propuesta de paradigma estilístico contrario a los modelos herreriano y gongorino, expuesto en su prólogo.

Se impone, por lo tanto, cuestionar lá unidad de las poesías de La Torre y el apéndice y la interpretación del volumen como muestra de una actividad literaria colectiva, en contra de lo que estima Cerrón Puga: «Fuera o no fuera el poeta de Salamanca, es indudable que a su escuela poética pertenece; así lo demuestra la propia estructura de la edición que nos ha legado Quevedo, la cual es algo más que la recopilación poética de un solo autor pues en ella, como sabemos, se incluye un apéndice con traducciones del Brocense, de Almeida, de Alonso de Espinosa y de fray Luis de León» (1984: 50). Para la estudiosa, el apéndice no puede considerarse un mero añadido, unos preliminares o un prólogo, sino «parte integrante con pleno derecho de un volumen poético que aspiraba a convertirse en remedo de las antologías petrarquistas italianas» (1984: 51). Pero este designio fue alterado por Quevedo al otorgar a La Torre un protagonismo autorial que en el plan anterior compartía con el Brocense.

La información que se desprende de la licencia del Archivo de Simancas permite matizar y corregir estas conclusiones. La Torre debió mantener vínculos literarios e intelectuales con los poetas de Salamanca, porque al menos en 1588 aparece avecindado en esta ciudad. Pero su relación no cristalizó en una antología colectiva, ya que por estas fechas el autor preparó la publicación de su obra individual, sin añadidos. La edición de 1631 modifica en cierto sentido la voluntad autorial al incorporar, aunque solapados, una serie de textos que otorgan al volumen la engañosa apariencia de rimas de varios.

Cada una de estas dos secciones emana de concepciones y criterios poéticos totalmente dispares, que determinan que su conjunción sea forza-

\footnotetext{
${ }^{11}$ Blanco (1982: 338) sopesa la posibilidad de que «las tres partes del Apéndice hubieran formado anteriormente una más densa unidad, con más cohesión, más completa, quizá todo un prólogo, que alguien quitó en su totalidad de donde estaba y colocó en partes al final».

${ }^{12}$ Cfr. Marsá, 1999: 191-192; Simón Díaz, 2000: 122-149; Marsá, 2001: 56-57.
} 
da y artificiosa. Las poesías de La Torre forman una unidad en sí misma, en la que las formas poéticas determinan la división en libros, en contraste con el anárquico engarce de traducciones y pasajes en prosa, de diversa y discutible autoría, amalgamados en el apéndice ${ }^{13}$. Si el primer conjunto brinda una muestra de imitaciones, con diverso grado de creatividad, el segundo se encierra dentro de las modestas pretensiones de la traducción ${ }^{14}$.

Hasta el momento, el apéndice ha reportado la prueba sobre la que se asienta la pertenencia de La Torre al círculo de Salamanca, corroborada por la intervención activa de Juan de Almeida y de Sánchez de las Brozas en el proyecto editorial que incluía como primera parte las Obras. Almeida, señero representante de los reformadores salmantinos ${ }^{15}$, mantiene con el Brocense una amistad que justificaría el intercambio de traducciones y, sobre todo, la entrega a este del manuscrito de las poesías de Francisco de la Torre antes de 1572 (Cerrón, 1984: 56-57). A partir de entonces, Sánchez se ocupa de los trámites previos a la impresión: «en 1580, o más tarde, todo el volumen con las poesías del Brocense incluidas sería presentado a la aprobación de Ercilla, presumiblemente por el propio Sánchez» (Cerrón, 1984: 57). La edición no se llevaría a cabo porque por aquellas fechas Almeida, el Brocense y, en general, el grupo de humanistas salmantinos estaban en entredicho, por sus innegables vínculos con el ramismo (Blanco, 1982: 74-79; Cerrón, 1984: 57-58).

El manuscrito con la poesía de La Torre y las traducciones llega de alguna forma a Quevedo, que decide publicarlo. Blanco (1982: 343-352) y

${ }^{13}$ Todas estas anomalías han sido notadas por Coster (1925: 107), Sena (1974: 11 20), Blanco Sánchez (1982: 335-385), Hughes (1982: 10-11). Entre ellas destacan los títulos de Catedrático y Maestro dados al Brocense, la presentación de Ariosto y Fray Luis como contemporáneos, la mención de «tres tan grandes poetas» (fol. 141v) por parte de uno de ellos, la estructura abrupta y con divisiones injustificadas.

${ }^{14}$ Resulta inexacto considerar las poesías de La Torre como «otro ejercicio de traducción paralelo al del llamado apéndice; traducción que, es verdad, acaba por levantar el vuelo y convertirse en reelaboración original de los modelos aceptados como clásicos, pero que no por eso deja de inscribirse en el marco de la imitatio» (Cerrón, 1984: 52).

${ }^{15}$ Las propuestas de identificación de este personaje comienzan con Fernández-Guerra (1860: 90), que le atribuye origen portugués y el sobrenombre de «El Sabio». Coster (1925: 102-104) no admite que ese Almeida sea el Rector de Salamanca, Maestro en Teología, que preparó la edición de Francisco de la Torre. Crawford (1927: 369) implica a los dos personajes en el proyecto editorial de las Obras: el Rector, que traduce la oda de Horacio, y su homónimo más joven, que añade al manuscrito de La Torre las traducciones y se encarga de solicitar la aprobación entre 1579 y 1591. Sena (1974: 6977) y Blanco (1982: 55-79) ofrecen, con los datos biográficos, la edición de sus textos: transcripción paleográfica, seguida de lectura moderna (Sena, 1974: 195-311) y edición anotada, con posibles atribuciones (Blanco, 1982: 525-700). Blanco asimismo lo relaciona con el ramismo, corriente de signo anti-aristotélico representada por Pedro Ramús o Pierre de la Ramée (1982: 74-79). 
Cerrón (1984: 58-65) reconstruyen una trayectoria que sigue un curso paralelo a la edición quevediana de Fray Luis de León. De hecho, ambas obras obtienen la licencia el 14 de marzo de 1630, después de haber superado las aprobaciones de los mismos censores, y se publican el mismo año. La portada de las Obras propias y traducciones... de Fray Luis de León constata la procedencia del original: «SACADAS DE LA LIBRERIA / de don Manuel Sarmiento de Mendoça, / Canonigo de la Magistral de la santa / Iglesia de Seuilla» (fol. 1r). En la primera dedicatoria, al propio Sarmiento, Quevedo declara haber recibido del canónigo el original ahora publicado: «Dexome V. M. estas obras grandes en estas palabras doctas, y estudiadas, para que siruiessen de antidoto...» (fol. $5 r-v$ ).

De este dato se ha inferido que la poesía de La Torre llegó a Quevedo por idéntico conducto y que ambos originales procedían de la biblioteca del Brocense. Para Cerrón (1984: 61-65), Sarmiento los adquiere al cumplir el encargo del Santo Oficio de trasladar los libros del Brocense de Salamanca a Valladolid en 1600 y en 1624 se los entrega a Quevedo en Sevilla ${ }^{16}$. El cotejo de los inventarios de los libros del Brocense, en 1600 y 1602, apoya la suposición, si se acepta que uno de los legajos desaparecidos en el segundo incluiría las poesías de La Torre.

Blanco y Cerrón trazan una común trayectoria de los originales aplicando a La Torre una evidencia que sólo se puede probar para Fray Luis, la intervención de Sarmiento de Mendoza ${ }^{17}$. Algunos de los pasos previos del razonamiento que da lugar a esta teoría admiten ser cuestionados:

- El manuscrito de las poesías de La Torre no perteneció a Almeida, que muere antes de que se inicien las diligencias de impresión por parte del propio autor.

- El Brocense no solicitó la autorización para imprimir, sino Francisco de la Torre, encargado de los trámites legales del primer proyecto editorial.

- Si se admite que Sánchez tuvo en su poder el manuscrito, esto ocurriría después de 1588.

- Que la biblioteca de Sánchez custodiaba los manuscritos de Luis de León y de Francisco de la Torre es en ambos casos una posibilidad que queda por demostrar.

${ }^{16}$ En 1624 Quevedo, con ocasión de su viaje a Cádiz formando parte de la expedición real contra Carlos Estuardo, pasa por Sevilla y allí coincide con Manuel Sarmiento de Mendoza (Astrana Marín, Vida turbulenta de Quevedo. Madrid, 1945. 351-352. En Cerrón, 1984: 64, n. 195).

17 Esta reconstrucción carece de base, como acertadamente razona Alatorre: «Si Quevedo no indica la procedencia «exacta» del manuscrito, la explicación lógica es que no la conocía. (...) no se ve por qué en el caso de fray Luis explica Quevedo la proveniencia del manuscrito mientras en el de Torre se esfuerza por echarle tierra al asunto» (Alatorre, 1999: 68). 
- Quevedo obtuvo de Sarmiento el original luisiano, pero sólo por conjeturas se puede extraer que el canónigo sevillano le proporcionase también el de La Torre.

En sus prólogos, Quevedo constata la afinidad del autor por él editado y Herrera, al que acusa de apropiación de palabras, dicciones y rasgos de estilo $^{18}$, oponiendo la depuración idiomática de La Torre a los deslices de su imitador, más censurables en la edición de Pacheco (1619) que en el volumen de las rimas preparado por el sevillano (1582). Para Quevedo, la dirección de la influencia no ofrece dudas: Herrera «tuuo por maestro, y exemplo a Francisco de la Torre, imitando su diccion, y tomando sus frasis, y vozes tan frequente, que puedo escusar el señalarlas; pues quien los leyere, vera, que no son semejantes, sino vno» (fol. $11 r$-v) ${ }^{19}$.

El criterio y la autoridad de Quevedo legaban para la crítica posterior el problema de un parentesco literario difícil de probar. Secundaría su propuesta, entre otros, Coster (1925: 87-101), que defiende la prioridad de La Torre después de considerar los sonetos 17, 16, 19, 20 y 14 del libro II de sus poesías en relación con los versos herrerianos. Tras analizar léxico y estrofas, descarta la posibilidad de que se trate del mismo autor. Hughes (1982: 105) advierte en ambos poetas una común propensión a las innovaciones de lengua y estilo, que anticipan el movimiento cultista, a pesar de que Gracián sólo incluye en su Agudeza y arte de ingenio una cita de Francisco de la Torre, a propósito de la canción de la Tórtola solitaria $(\mathrm{I}-1)^{20}$.

Para Cerrón Puga, el parecido que manifiestan entre sí algunas composiciones de estos poetas denota una evidente relación en la que erige como modelo a Francisco de la Torre ${ }^{21}$, tomando como límite cronológico de escritura el año de la muerte de Almeida, 1572. Aunque las poesías de Herrera seleccionadas no pueden datarse con precisión, al menos la redac-

\footnotetext{
${ }^{18}$ El ejemplar anotado por Quevedo de la edición herreriana de Pacheco, que se conserva en el Seminario Diocesano de Vitoria, contiene la siguiente observación, intercalada al prefacio de Rioja: «De Torre primero lo aprendió». Según Komanecky (1975), las notas de este ejemplar confirman la interpretación de los preliminares a la poesía de La Torre como un ataque contra Herrera y la edición de Pachecho de 1619. Cfr. Azaustre, 2003.

19 Aunque las hojas de los preliminares carecen de foliación, aquí, y en referencias anteriores, se han numerado para una mejor localización de las citas.

${ }^{20} \mathrm{La}$ primera estrofa de esta canción es aducida en el discurso sobre la agudeza de improporción y disonancia, para ilustrar cómo la variedad de tiempos pondera la contrariedad de las circunstancias, pasando de la improporción del bien pasado con el dolor presente a la proporción entre dos recíprocas penas (Gracián, 1980, 1: 78).

${ }^{21}$ Cerrón relaciona once composiciones de La Torre, en su mayor parte sonetos, con catorce de Herrera (1984: 78, n. 246).
} 
ción de dos de ellas ocurriría en torno a $1577^{22}$. De su razonamiento cronológico infiere las siguientes conclusiones: «mientras que tenemos constancia de que las Obras de Francisco de la Torre son anteriores a 1572 (fecha de la muerte de Almeida), de ninguna de las poesías de las que de Herrera nos interesan puede decirse lo mismo ya que sólo dos pueden fecharse con una cierta precisión» (1984: 79). Tal aserto, basado en la discutible premisa de que la falta de datos implica posterioridad, pierde consistencia a la luz del hallazgo expuesto en este trabajo, la autorización de 1588 , que anula el valor referencial del año $1572^{23}$.

Francisco de la Torre obtenía permiso para imprimir sus poesías seis años después de la publicación de Algunas obras (1582). De los catorce poemas de Herrera que proporciona Cerrón (1984: 78, n. 246), ocho pertenecen a este volumen ${ }^{24}$ :

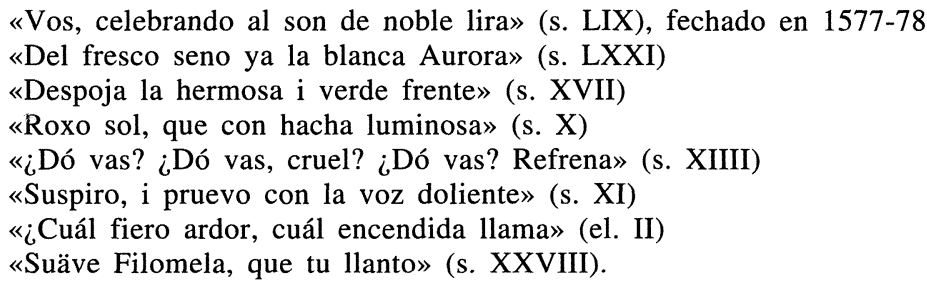

Se corresponden respectivamente con las siguientes composiciones de Francisco de la Torre:

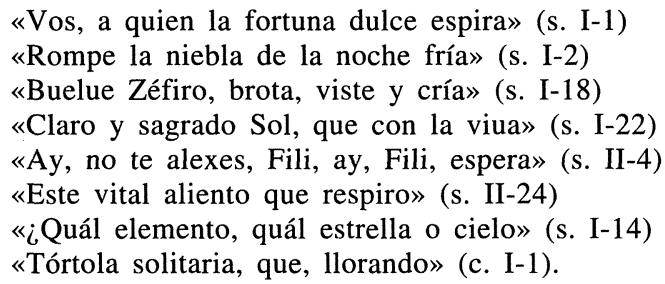

De esta lista, la elegía II y el soneto XXVIII de Herrera, por una parte, y el soneto I-14 y la canción I-1 de La Torre mantienen una vaga semejanza, que hace cuestionable cualquier filiación. En tres casos puede

${ }^{22}$ Se trata de los sonetos Vos, celebrando al son de noble lira, dedicado a Barahora de Soto, que no sería anterior a 1577 ó 1578, y El oro crespo al aura desparzido, publicado en las Flores de baria poesía. México, 1577.

${ }^{23}$ El Francisco de la Torre Escobar, natural de Santa Fe de Bogotá, propuesto por Alatorre, no comenzaría a escribir antes de 1577 , de forma que podría haber conocido la edición de la poesía de Herrera de 1582 (Alatorre, 1999: 44-53).

${ }^{24}$ Algunas obras de Fernando de Herrera. Sevilla: Andrea Pescioni, 1582. En Cuevas, 1985: 347-469. 
proponerse una fuente italiana común, que la propia editora indica en las notas que acompañan al texto poético de los sonetos I-1, I-2 y II-4, imitaciones de Molza, Petrarca y Varchi, respectivamente (Cerrón, 1993: 73, 75, 136). La selección, por lo tanto, quedaría reducida a los sonetos LXXI, X y XI de Herrera y los sonetos I-2, I-22 y II-24 de La Torre.

Tres de los ejemplos han sido extraídos del volumen de los Versos ${ }^{25}$ preparado por Pachecho (1619):

«Pues cubre al orbe'n assombrado velo» (s. LXXV, 1. III)

«Cuán bien, oscura noche, al dolor mío» (s. LXV, 1. II)

«La Idra d'amoroso pensamiento» (s. LXXIV, l. II).

Cerrón relaciona los dos primeros con el soneto I-20 de La Torre y el segundo con el soneto I-30:

«iQuántas vezes te me has engalanado» (s. I-20)

«Esta zelosa hydra, que en mí siento» (s. I-30).

Las restantes composiciones proceden de distintos testimonios para los que existen fechas probables:

«Çumeta, vuestra noble y dulçe lira» (ms. B, 1578) ${ }^{26}$

«Los ojos bellos y las varias flores» (ms. B, 1578)

«El oro crespo al aura desparzido» $(1577)^{27}$.

El primero remite al soneto inicial de las Obras de La Torre, imitación de Molza: «Vos, a quien la fortuna dulce espira», y los dos siguientes al soneto II-23: «La blanca nieue y la purpúrea rosa», basado en un soneto de Amalteo.

A la vista de esta relación, de las catorce composiciones herrerianas que pudieron haberse inspirado en la poesía de La Torre pueden ser excluidas la elegía II y el soneto XXVIII de 1582 y el soneto LXXV de 1619, por ofrecer sólo un lejano parecido con los correspondientes modelos propuestos. Tampoco se puede postular una relación genética cuando la probable fuente italiana común está documentada, tal como ocurre en cuatro poemas de Francisco de la Torre y en seis de Herrera ${ }^{28}$. Quedarían,

${ }^{25}$ Versos de Fernando de Herrera. Ed. de Pacheco. Sevilla: Gabriel Ramos Vejarano, 1619. En Cuevas, 1985: 471-818.

${ }^{26}$ Aunque la sección del manuscrito B $(10.195$, BNM) dedicada a Herrera está fechada en 1578, Cuevas (1985: 255 n.) propone 1572, año del fallecimiento del Duque de Alcalá recordado en el soneto.

27 En el ms. 2973 de la BNM, Flores de Baria Poesia. México, 1577.

${ }^{28}$ El soneto I-1 de La Torre, imitación de Molza, es puesto en relación con Vos, celebrando al son de noble lira y Çumeta, vuestra noble y dulçe lira; el soneto I-18, inspirado en Petrarca, con Despoja la hermosa $i$ verde frente; el soneto II-4, que se basa 
por lo tanto, cinco piezas herrerianas que, en diferente, grado se asemejan a otras tantas de La Torre. En este caso, se impone la difícil tarea de resolver la dirección de las influencias.

No conviene pasar por alto que los datos oficiales relativos a las diligencias administrativas y trámites de impresión no implican una determinada fecha de escritura. Se superpone, además, la probabilidad de una difusión manuscrita muy anterior al momento en que el texto se da por fin a la estampa y esta constatación es aplicable tanto a Herrera como a La Torre. Siguiendo un criterio cuantitativo, los datos cronológicos se inclinan a favor de Herrera, cuyos poemas se pueden datar en 1572, 1577 y 1582, mientras que para La Torre no se documenta ninguna fecha anterior a 1588. Con esto no se intenta presentar a Herrera como poeta más antiguo y, por lo tanto, imitado, sino simplemente exponer que la prioridad de La Torre no es demostrable y se funda 'en datos erróneos. A partir de ahora, debe retrasarse su ubicación temporal en al menos una década.

Para Cerrón, Quevedo no se limita a señalar las deudas poéticas que Herrera contrae con La Torre, sino que además lo identifica con el ingenio mendigo culpable de la ocultación del manuscrito, tras advertir en la palabra borrones una acepción estilística que aludiría a sus plagios, caracterizados por la oscuridad ${ }^{29}$. De este modo, la estudiosa establece entre el primer prólogo, a Ramiro Felipe de Guzmán, y el segundo, al lector, una conexión que dista de estar clara, pues en el primer texto Quevedo denuncia el hurto, llevado a cabo por algún envidioso, y las tachaduras del nombre del autor (fol. $8 r$ ) y en el segundo detecta los elementos que Herrera toma de La Torre (fol. $11 r-v$ ), sin que nada pruebe su voluntad de equiparar los borrones de un lugar con las imitaciones del otro.

El sevillano, según esta misma autora, accedió al manuscrito a través de su maestro Mal Lara, contertulio del Brocense, o bien por mediación de Ercilla, aprobador de su volumen poético de 1582 y de las obras de La Torre (Cerrón, 1984: 82). Cualquiera de estas vías alternativas admite objeciones. Si Herrera tuvo en su poder el manuscrito original de las poesías de La Torre, esto debió ocurrir después de 1588. Juan de Mal Lara no pudo proporcionárselo, ya que muere en 1571, cuando el original, si para entonces ya existía, pertenecía a su propio autor. Por otra parte, el Brocense no desempeñó ese protagonismo que se le ha atribuido en la preparación y custodia del manuscrito. Carece de sentido, por otra parte, implicar a Ercilla

en Varchi, con ¿Dó vas? ¿Dó vas, cruel? ¿Dó vas? Refrena, y el soneto II-23, versión de Amalteo, con Los ojos bellos y las varias flores y El oro crespo al aura desparzido.

29 «yo pienso que cuando Quevedo habla de los famosos «borrones» con los que se tachó cinco veces el nombre de Francisco de la Torre puede estar refiriéndose a borrones estilísticos (...). Esta puede ser la clave: los borrones serían las imitaciones que Herrera hizo de las poesías de Francisco de la Torre y esto, más el silencio en el que envuelve el hecho, es lo que Quevedo le reprocha» (Cerrón, 1984: 82). 
como intermediario y colegir de su cargo de aprobador vínculos de amistad con los autores sometidos a su juicio, ni el acceso de estos a las obras ajenas pendientes de aprobación.

Diversas suposiciones han tergiversado la genuina interpretación de la poesía de Francisco de la Torre en el marco literario de su época. La licencia de impresión fechada en 1588 permite avanzar un paso más en la difícil tarea de descifrar este enigma, para demostrar que el autor preparó el manuscrito de sus poesías con el propósito de publicarlas como creación individual. La referencia cronológica ahora aportada obliga a replantear el puesto generacional de La Torre y a cuestionar sus vínculos con otros poetas contemporáneos, supuestamente implicados en el proyecto editorial o la imitación de sus poesías.

\section{BIBLIOGRAFÍA}

AlatorRe, Antonio, «Francisco de la Torre y su muy probable patria: Santa Fe de Bogotá», Nueva Revista de Filología Hispánica 47, 1 (1999): 33-72.

AZaustre Galiana, Antonio, Preliminares literarios a las "Obras» del Bachiller Francisco de la Torre, en A. Rey, ed. Obras completas en prosa de Quevedo, vol. 1, t. 1. Madrid: Castalia, NBEC, 2003, 165-168, 171.

Blanco SÁNCHEZ, Antonio, Entre Fray Luis y Quevedo. En busca de Francisco de la Torre, Salamanca: Atlas, 1982.

CERRÓN PUgA, M. Luisa, El poeta perdido: aproximación a Francisco de la Torre, Pisa: Giardini Editori e Stampatori, 1984.

—, ed. Poesía completa de Francisco de la Torre. Madrid: Cátedra, 1993 (1984, 1. ${ }^{\text {a }}$ ed.).

COLÓN CALDERÓN, Isabel, «Sobre la edición perdida de Francisco de la Torre», Dicenda 20 (2002): 29-38.

COSTER, M. Adolphe, «Sur Francisco de la Torre», Revue Hispanique 65 (1925): 74-133.

CRAWFORD, J. P. Wickersham, «Francisco de la Torre and Juan de Almeida», Modern Language Notes 42, 6 (1927): 365-371.

Cuevas, Cristóbal, ed., Poesía castellana original completa de Fernando de Herrera, Madrid: Cátedra, 1985.

FERNÁNDEZ GuERRA, Aureliano, «Discurso de recepción en la Real Academia de la Lengua» (1857), en Discursos leídos en las recepciones públicas que ha celebrado desde 1847 la Real Academia Española, vol. 2, Madrid, 1860, 79-104.

GrACIÁN, Baltasar, Agudeza y arte de ingenio, Ed. Evaristo Correa Calderón, 2 vols. Madrid: Castalia, 1980.

HughES, Gethin, The Poetry of Francisco de la Torre, Toronto-Buffalo-London: University of Toronto Press, 1982.

KOMANECKY, Peter M., «Quevedo's Notes on Herrera: The Involvement of Francisco de la Torre in the Controversy over Góngora», Bulletin of Hispanic Studies 52 (1975): 123-133.

LEÓN, Fr. Luis de, Obras propias y Tradvciones Latinas, Griegas, y Italianas... Dalas a la Impression don Francisco de Quebedo Villegas... Madrid: Imprenta del Reyno, 1631.

MARSÁ VILA, María, El fondo antiguo en la biblioteca, Gijón: Ediciones Trea, 1999.

—, La imprenta en los Siglos de Oro, Madrid: Ediciones del Laberinto, 2001. 
MenÉndez Pelayo, Marcelino, Horacio en España, t. 1, Madrid: Imprenta de A. Pérez Dubrull, 1885.

PÉREZ-ABADín BARRo, Soledad, «Un nuevo dato sobre Francisco de la Torre: la real provisión documentada en el Archivo de Simancas», Bulletin Hispanique 2 (2003).

-, «Nota bibliográfica acerca de Francisco de la Torre», Rilce 19, 2 (2003).

—, «La poesía de Francisco de la Torre: un proyecto editorial frustrado», Criticón, 2004 (en prensa).

RoJo VEGA, Anastasio, «Manuscritos y problemas de edición en el siglo XVI», Castilla. Estudios de Literatura 19 (1994): 129-157.

SENA, Jorge de, Francisco de la Torre e D. João de Almeida, Paris: Fundação Calouste Gulbenkian-Centro Cultural Português, 1974.

SIMÓN DíAZ, José, El libro español antiguo. Análisis de su estructura, Madrid: Ollero \& Ramos, 2000 (Kassel: Edition Reichenberger, 1983, 1. ${ }^{a}$ ed.).

TORRE, Francisco de la, Obras del Bachiller Francisco de la Torre. Dalas a la impression D. Francisco de Queuedo Villegas... Madrid: Imprenta del Reyno, 1631.

Zamora Vicente, Alonso, ed., Poesías de Francisco de la Torre, Madrid: Espasa-Calpe, 1969 (1944, 1. ${ }^{\mathrm{a}}$ ed. $)$. 


\title{
RESUMEN
}

Al margen de las Obras de Francisco de la Torre, por Soledad PérezAbadín Barro.

Las Obras de Francisco de la Torre, publicadas por Quevedo en 1631, incluyen una segunda parte, que más tarde se llamaría «apéndice», con traducciones ajenas al proyecto inicial preparado por el propio autor décadas antes. El sumario de la licencia de impresión de 1588 describe el contenido de la obra aprobada como «los versos líricos y adónicos y la bucólica», sin mencionar las traducciones. La nueva fecha lleva a cuestionar el protagonismo de Almeida y el Brocense en la preparación y transmisión del manuscrito que más tarde llegaría a Quevedo. El volumen de 1631, por lo tanto, no puede considerarse como un exponente de un ejercicio poético colectivo del círculo salmantino. Los datos cronológicos también permiten replantear la relación del autor con Fernando de Herrera.

Palabras clave: apéndice, traducciones, Francisco de la Torre, Juan de Almeida, Sánchez de las Brozas, Fernando de Herrera.,

\begin{abstract}
Francisco de la Torre's Works, published by Quevedo in 1631, enclose a second part, described in later years as an "appendix», with translations that did not belong in the original project prepared by the author some decades before. The summary of the 1588 license for printing describes the work's content as «lyric and adonic verse, and bucolic», without mentioning the translations. The new date throws into question the participation of Almeida and Brocense in the preparation and transmission of the manuscript that came to Quevedo. Therefore, the volume of 1631 cannot be considered as an example of salmantin group's collective poetical essay. Related chronological considerations also throw new light on the relationship between the author and Fernando de Herrera.
\end{abstract}

Key words: appendix, translations, Francisco de la Torre, Juan de Almeida, Sánchez de las Brozas, Fernando de Herrera. 